\title{
Response of biotester Lepidium sativum to the effects of native herb extracts and phytoinvader Solidago canadensis
}

\author{
Yulia Zagurskaya*, Vladimir Ufimtsev \\ Federal Research Center of Coal and Coal Chemistry SB RAS, 10 Leningradsky avenu, Kemerovo, \\ 650065, Russia
}

\begin{abstract}
The effects of aqueous extracts from aboveground parts of Solidago canadensis L. as well as from dominant species of four main succession stages on the degraded forest soils of the Kemerovo region (Filipendula ulmaria, Chamaenerion angustifolium, Cirsium setosum, Poa pratensis) on the germinating capacity and speed of Lepidium sativum seeds were investigated. It was showed that Solidago canadensis and Filipendula ulmaria have the greatest effect on the reduction of germinating capacity of Lepidium sativum seeds.
\end{abstract}

\section{Introduction}

According to a number of scientific reports, Solidago canadensis can suppress the development of many herbs on the different stages of ontogenesis [1 - 5] leading to the dysfunction of native phytocenosis and failure of disturbed land restoration caused by S. canadensis invasion beyond their native areal (North America) [6 - 10].

To study the effects of Solidago canadensis L. on the germinating capacity of other plants' seeds and to compare the obtained effects with ones of dominant species of four main succession stages on the degraded forest soils of the Kemerovo region (Filipendula ulmaria, Chamaenerion angustifolium, Cirsium setosum, Poa pratensis) we selected Lepidium sativum L. (cress) characterized by the most balanced differentiating ability in assessing the allelopathic activity of various "donor plants" [11].

\section{Materials and Methods}

Seeds of biotester Lepidium sativum L. were couched under exposure to the aqueous extracts from the aboveground parts of five herbs with a different life strategy.

\footnotetext{
*Corresponding author: syjil@mail.ru
} 


\subsection{Object of study}

Testing culture: seeds of Lepidium sativum L. (cress). Aqueous extracts were obtained from crushed dry aboveground parts (the competitiveness of herbs decreases in a row during the development of disturbed ecotopes):

1. Solidago canadensis L. (Canadian goldenrod) - potentially invasive species;

2. Filipendula ulmaria (L.) Maxim. (meadowsweet) - dominant of climax cenosis;

3. Chamaenerion angustifolium (L.) Scop. (willow-herb) - dominant of post-fire upland cenosis;

4. Cirsium arvense var. integrifolium Wimm. \& Grab. = Cirsium setosum (Willd.) Besser ex M.Bieb. (yellow thistle) - dominant of early successive fallow cenosis;

5. Poa pratensis L. (rough-stalked bluegrass) - dominant of meadow cenosis.

\subsection{Methods}

As a control, distillated water was used. All experiments were performed in triplicate. Generally, 100 seeds were investigated. Germinated seeds were counted daily following the day when the experiment was started. As the samples drying, the new appropriate extracts were added.

Germination capacity and speed of germination were determined at the different stages of the experiment according to the State Standard No. 12038-84 [12]; total germination energy (GE) of seed per day was calculated [13].

\subsection{Statistics}

Statistical analysis was performed by Microsoft Excel software using the graphical analysis for minute samples characterized by a different distribution.

\section{Results and discussion}

The maximum germination period for L. sativum seeds was 5 days including the day as the experiment was started. The germination capacity and speed $(19 \%$ on the first day of the experiment and about $70 \%$ in total) was discovered in the control group; the extracts obtained from all studying herbs at the first stage of the experiment were associated with significantly reduced germination (Fig. 1). At the same time, the majority of studied extracts lose their effects after third day of the experiment - the viability of seeds of testing culture approach the control values excluding the extracts obtained from Solidago canadensis and Filipendula ulmaria associated with the suppressed development of testing culture. Moreover, Filipendula ulmaria was associated with three-fold decreasing of germination capacity compared to the control, while potentially invasive Solidago canadensis - with only one third one. Generally, average coefficients of germination capacity are equal to the dynamics of germ appearance (Fig. 2). The germination rate of Lepidium sativum seeds reflecting the germination speed at a certain stage of the experiment was decreased in the majority of the studied extracts excluding Poa pratensis, but the overall germination capacity for the most of variants was equal to the control. 


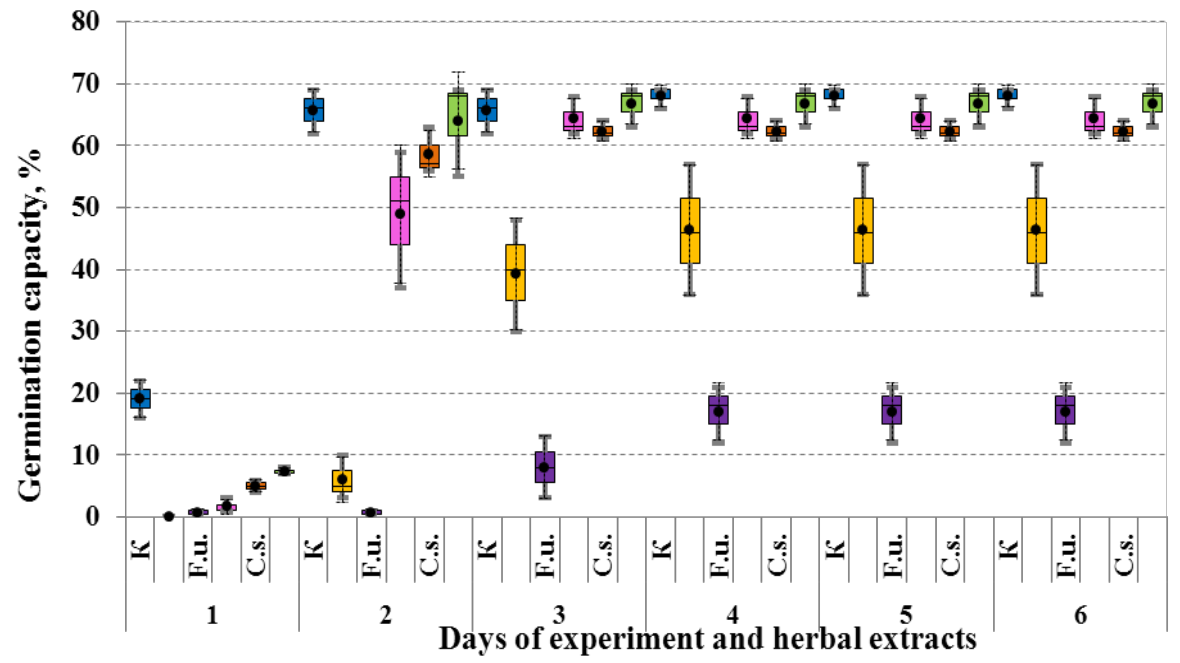

Fig. 1. Dynamics of germination of Lepidium sativum seeds under exposure to the extracts from the herbs with a different life strategy: box -1 and 3 quartile, a line in the center of the box - median, error bars - minimum and maximum value, point - mean, error bars (thin line) - standard deviation; here and after: $\mathrm{K}$ - control (distillated water), S.c. - Solidago canadensis, F.u. - Filipendula ulmaria, Ch.a. - Chamaenerion angustifolium, C.s. - Cirsium setosum, P.p. - Poa pratensis.

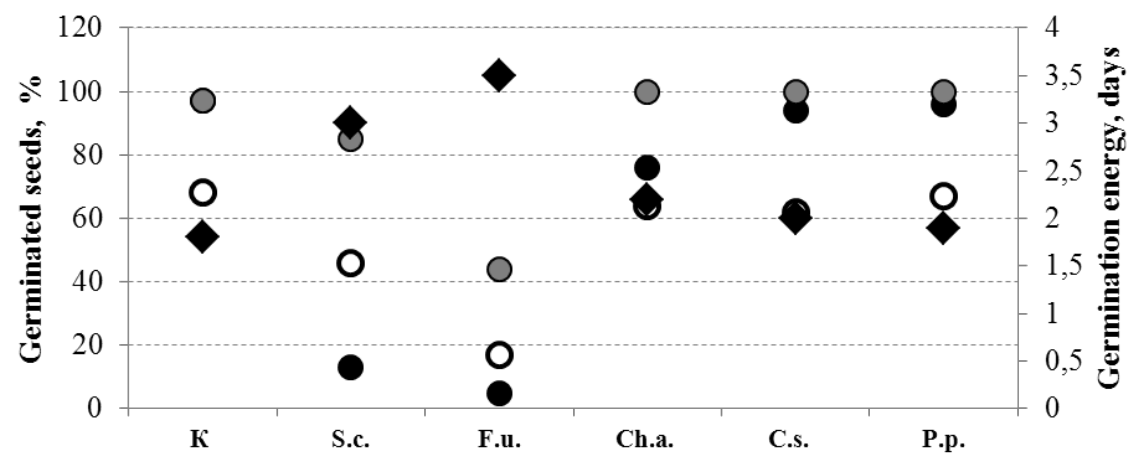

Herbal extracts

OGermination capacity (\%)

o Germination rate $2 * *(\%)$
- Germination rate $1 *(\%)$

- Germination energy (days)

Fig. 2. Germination of Lepidium sativum seeds under exposure to the extracts from the herbs with a different life strategy: * rate of germinated seeds at the second day of the experiment (\% of germinating seeds), $* *$ of germinated seeds at the third day of the experiment ( $\%$ of germinating seeds).

Germination energy (GE) of seeds including moments as each part of germ appeared. An increased GE reflects the delay in seeds' germination. In our experiment, the maximum GE values after exposure to Filipendula ulmaria and Solidago canadensis extracts (3.5 and 3 days, respectively) were significantly different from other samples (about 2 days). 


\section{Conclusion}

It was shown that aqueous extracts from the aboveground parts of Cirsium setosum and Poa pratensis have no effects on the germination capacity of Lepidium sativum seeds compared to the control. Chamaenerion angustifolium can suppress the germination in the first days but then an inhibitory effect disappears. The most pronounced effect on the germination capacity of $L$. sativum seeds was showed for Solidago canadensis and Filipendula ulmaria.

We are particularly indebted to N.V. Burova and A.V. Stolyarov for their conscientious determination of germination capacity. This work was supported by the research project of the Kuzbass Botanical Garden, Federal Research Center of Coal and Coal Chemistry SB RAS No. 02862021-0010 in the framework of the State Task No. AAAA-A21-121011590010-5.

\section{References}

1. G. Chen, Ch. Zhang, L. Ma, Sh. Qiang, J. A. Silander, L. L. Qi, J. Integrat. Agric. 12(5), 835-845(2013)

2. A. Fenesi, C. I. Vágási, M. Beldean, R. Földesi, L.-P. Kolcsárb, J. T. Shapiro, E. Török, A. Kovács-Hostyánszkie, Basic Appl. Ecol. 16, 335-346 (2015)

3. A.P. Gusev Rus. J. Biol. Invas. 6(2), 74-77 (2015)

4. V.M. Shmelev, A.N. Pankrushina, V. Tver SU 3(55), 130-135 (2019)

5. C. Wang, B. Wu, K. Jiang, J. Zhou, D. Du, Urban. For. Urban. Gree. 38, 145-156 (2019)

6. B. Tokarska-Guzik, B. Węgrzynek, A. Urbisz, A. Urbisz, T. Nowak, K. Bzdęga, Biodiv. Res. Conserv. 19, 33-54 (2010)

7. Black book of the flora of Siberia (Novosibirsk, "Geo" Publ., 2016), 440

8. Yu.K. Vinogradova, S.R. Mayorov, L.V. Khorun, Black data book of the flora of Central Russia (Moscow, Geos, 2010)

9. M. Stefanowicz, M. Stanek, M. L. Majewska, M. Nobis, S. Zubek, Appl. Soil Ecol. 136, 168-177 (2019)

10. M. Richardson, P. Pyšek, M. Rejmánek, M. G. Barbour, F. D. Panetta, C. J. Divers, Distrib. 6(2), P. 93-107 (2000)

11. A.F. Buharov, D.N. Baleev, RUDN J. Agro. Animal Ind. 1, 32-39 (2012)

12. GOST $R$ 12038-84 Agricultural seeds. Methods for determination of germination. (1986)

13. Agricultural dictionary reference (Moscow, Leningrad, Selkhozgiz, 1934) 